\title{
Index des lieux et des matières
}

abbaye, voir aussi roi de l'abbaye 11,17 , 132,139

abbé 11, 17, 71, 103, 131-132, 136, 139, 164

- de la jeunesse (Beaucaire) 17

- de Lescache (Cambrai) 136

- des conards (Rouen) 17

- des malprofitants 150

Abbeville 17, 25, 39-40 ${ }^{88}, 89$

académies littéraires 25

administration $20,35,47,65,73,79,121$, 162

Aix-en-Provence 17

Allemagne $10,15,20^{+33}, 30,43,96^{4}, 106$, 159

alliances interrégionales 95, 103

Alsace $95^{+3}, 98,100,103-104,107^{33}-108$, $110-111$

amendes $14-15,38,87^{+33}, 115,117,149$

Amiens 25, 26, 34, 83, 86

amoureuse vie, roi de l', voir roi des ribauds

Amtleute (officiers) 96, 98

Angleterre 24, 30, 32-33 $3^{51}, 36-37,39-42$, $44,52-55^{+59}, 57,64^{5}, 67,69,71-74,76-$ $77,79,81^{9}, 159$

Anjou 14-15 $5^{+22}$

Arabes 155, 157

Arbre des batailles (traité d'Honoré

Bouvet) 70

arbitrage 15, 104-106

archers, voir roi des archers

Argovie 107, 111

armaturos, voir aussi office d'armes 32

armes, voir aussi roi d'armes $40,45,47^{20}$ $49^{30}, 51^{40}, 54^{+55}-58,77,84,110,118$, 150,163

armes, armoiries 9, 40, 53, 55-57, 66, 73$74,118-119,163$

armorial $16^{24}, 57,64-65^{7}, 73-74^{+61}, 75$

Arran 73

Arras 17, 25-26, 29, 34, 86-88, 90-91

arrêt du travail 104

Artois $15^{22}, 20^{34}, 41^{94}, 45-46,48,132,141$

assemblée $24,98^{9}-99^{+14}, 100-101^{20}, 103-$

$104,106^{+32}-108^{+38}, 109,112$

associations de métier 25-26, 38

Astene 133

aumône, voir roi de l'aumône autonomie 76,117

Autriche 35, 50

Auvergne 14-15

avocat $64,68^{25}, 73,113-117,150$

avocat légal 35

Bâle $95^{3}-96^{6}, 98^{9}, 106^{31}-107^{35}$, 109

barbiers, voir aussi roi des barbiers $95^{3}$

basoche, voir aussi roi de la basoche 10 , $113^{+1}-116^{+10}, 117-119,121,163$

- de la chambre des comptes 118

- du Châtelet 118

- du parlement 119

Beaujolais $15^{19}$

Beaucaire 17

Beauvais $39^{+87}, 40^{88}$

Bergues 133, 137

Berne 99-101

Berry $14-15^{+22}, 73,85$

Béthune 25

Bettelvoigt, voir prévôt de mendiants

Bordeaux 86

Bourgogne $15^{22}, 20^{34}, 32,35,37,39^{+84}, 43$, $46^{+12}-47,49-50,54^{56}-57,64^{5}, 70^{36}, 85-$ $86^{+29}, 90,100,148$

Bouvines $158^{15}$

Bouvines, bataille de 81,156

boycottage $98^{7}, 105-106,110^{+43}$

Brabant 27, 39, 45-46, 48, 138

brigands, voir aussi roi des brigands 82 $83,146,150$

Brisach $95^{3}, 100,108^{+38}$

Brisgovie $102^{25}, 110-111$

Bruges $35,46^{12}, 69,82^{+12}, 88-89,135$, 143

burlesque 131-132, 134-135, 137-138, $140-142,144$

Caen 25

Cambrai 25, 83, 86, 89, 136

Capoue 31

capitaine $17,51^{+39}, 150$

- de la basoche (Parlement de Paris) 117 118

- du Pinon (Douai) 136

carnaval $11-12,102^{25}, 118,131,133-134$

Castelnuovo 31

cercles (Kesslerkreise) 100 
cérémonies 9, 40-42, 55-56, 58, 64, 66$68^{25}, 71,76-77,115,118-119,131$, 163-164

chambre de rhétorique $132,136-144,162$ - de baume (Balsamflower) 143 chambre des comptes (Paris) 119, 149

Champagne $15^{22}, 24,39,42-43,46,49^{+30}$ charivari $17^{27}, 131,137$

chaudronniers, voir aussi roi des chaudronniers $95^{3}, 98,100-101^{+21}, 110-112$

Châtelet 119

Chauny 83

clercs, voir aussi roi des clercs 113-114, 116-119, 162-163

Cleomadès (roman d'Adenet le Roi) 2728

collège (des hérauts d'armes) $55-57,75$

Colmar $95^{3}, 102$

communauté de métier $113^{1}, 115$

compagnons, voir aussi roi des compagnons $31^{38}, 95^{+3}-96^{+6}, 98^{+9}, 100-104^{+29}$, $105-106^{+31}, 107^{+33,35}-110^{+43}, 111-112$, 153,164

comte (Graf) 20,36, 158

- des ménestrels (Spielgraf) $20^{33}$

- des ribauds 82,91

conards, abbé des, voir abbé des conards confiscation 14

confrérie $13,15,19,24-27,29,34-35$, $38-39,43,99^{+14}-100,108^{38}-109,112$, $162-163$

- des jongleurs et des bourgeois (Arras) 26,34

- urbaine 24

coq, voir roi du coq

coquillards 148-149, 152-153

coquille, voir aussi roi de la Coquille 148 149

Corbie $15^{22}, 46$

cordonniers $95^{3}-96,103-107^{+33}, 108^{+3}-$ $110,151^{25}$

couleuvriniers, voir roi des couleuvriniers cour, 10-11, 25, 27-29+23, 30-32, 35-40, $42-47,51-54,58,80-81,84-86,92$, $113^{3}, 159,161$

- des ducs Valois 32

- des miracles $12^{14}$

- ducale et royale $18,26,31,35-36,41-$ $43,85,125,157,163-164$

- princière $17,29-31,34,36,42-43,84$, 163-164

- seigneuriale $27,29,34,42$

cour, société de 42,157 couronne $11^{9}, 28,32-33,35^{+62,63}, 64-65$, 67-68, 74, 118-119, 126, 142, 153, 159, $161,163-164$

- d'épines, voir épines

- imperiale $67^{+18}$

couronnement $9,48^{24}, 64,67,71,76-77$

crime $74^{61}-75,84-85,92,146-148$

- monarchie du crime 151, 153

- organisations criminelles 146-147

Dauphiné $15^{19}, 18^{29}, 53$

Deinze 133

demoiselle noire (dignité parodique) 136

Danemark 69

dérision $10-12,14,90,147,153,161-162$

Dieppe 25

dignité 9,11-13,16-18, 21, 138, 142, $147,162-163$

- festive, voir aussi Forestier de Bruges; roi de l'Epinette 132-133, 135-137, 140

- parodique, voir abbé; demoiselle noire; droge jonckheere; princes; rois moqueurs

- royal 126,162

Dijon $39^{84}, 119,146,148$

distinction sociale 16,42

Dixmude 133

domestique $31-33,35-38,40^{89}, 43,85^{21}$

Douai $25,29,41,83^{16}, 86-87^{+32}, 88,91$, 136

doubles, voir roi de France

doyen (deken) 140-141

Dreikönigstag, voir Épiphanie

droge jonckheere (dignité parodique) 136

échecs, jeu d' 155-157, 159-160

- chah (pièces arabo-persanes) 157

- fou (pièce) 158-160

- reine (pièce) 157-160

- roc, voir aussi échecs, tour du jeu d' $158-160$

- roi, voir roi du jeu d'échec

- tour (pièce) 158, 160

- vizir (pièce) 157

écoliers, voir roi des écoliers

écoles des ménestrels 36, 39-40

Écosse 64, 66-76, 78-79, 163

écoutète (Schultheiss) 96, 98, 100-10120, 104, 106, 110

Édimbourg 70, 76

égalité, attitude égalitaire 140

Empire germanique, voir Allemagne 
épines, couronne d' (Corona spinea) 11, 164

Épinette, voir roi de l'Épinette

Épiphanie (Dreikönigstag) 10, 17, 124$125,127-129$

espion/espionnage 37,101

évêques $71,77,131,139,155,158,160$

- des fous (en général) 11, 18, 132-133, $135,144,164$

- des fous (Lille) 136

- des innocents 18, 133

féodalité

- guerre féodale 156

- mentalités féodales 156

- roi féodal 159

fête $13,16^{+24}, 18,21,34-35,39-40^{+89}, 41$, $46-47,55,58,71,88-89,93,96^{4}, 99$, $101-102,108^{38}, 111,115,118,121$, $131,133-139,141-142,144,163$

- des fous 11, 89, 133, 135, 138

- des Rois, voir aussi Épiphanie 111, 118-119, 161

- culture festive urbaine 133,139

fève, voir roi de la fève

Flandre $15^{22}, 16,20^{34}, 27,39,45-46,48$, $64^{3}, 69,82,91^{+55}, 131-138,141,143-$ 144

fonctions $11,12,17^{+27}-18,20^{+33}, 24,31$, $35,37-38,40,43-47,49-50,52,55$, $57^{71}-58,70,72-73,80-81,83-86,88-$ $90,93,96^{4}, 98,100,105-106,115-118$, $121,140-141,144,156,159^{16}-160,164$

forains 89

Forestier (dignité festive à Bruges) 89, 135-136

Forêt-Noire 95, 100, 111

forgerons $95,98,105,107^{+33}, 110,164$

fou/fous, voir aussi évêque; roi; fête des fous 138

France $9-11,14-15^{+22}, 17-18^{+28,29}, 20$, $24-25,29^{23}, 32,35^{+63}-39,42,44,46$, $48,53-55,57^{+67}, 66^{13}, 69-70^{36}, 71,80$ $81,84,88,90,131-132,139,142,144$, $157,163-164$

gages, voir aussi rémunérations 24,33 , $37-38,51^{39}, 85,117$

Gand 25, 133-134, 140-141, 143

Gesellschaft vom Esel, voir aussi sociétés nobiliaires 15

grand abbé, voir roi de l'abbaye gratifications, voir aussi rémunérations 34 , 37

gravamina majeurs 108

Grenoble 17, 119

Guise $86-87^{+32}, 89$

Haguenau $95^{3}, 98,100,109$

Hainaut $28-29,32,39,41^{94}-43,45-46$, 48-49

Heger (officier) 96, 98

hérauts d'armes, voir aussi office d'armes $15^{22}, 33^{51}, 44^{+1}, 47^{+22}, 53,55,58,78$

hiérarchie $19,52,54-55,64-65,74,76-$ $79,104,111,116,121,125-127,129$, 132-133, 136, 139-141, 148-149, 153, $158,162,164$

Hollande $29^{+23}-30,45,143$

huissier (Weibel) 100-101 ${ }^{20}, 104,106,110$

incendiaires 101

inversion $10^{+7}-12,14,18-19,93,126$, $133,142,162,164$

investiture, voir aussi couronnement; sacre 65-67, 71, 131-135, 137-140, 142, 144, 163

Italie $31,80-81^{8}, 88,155$

Jarretière, ordre de la 54, 69

jeunesse, abbé de la, voir abbé de la jeunesse

jeux-partis 25

joculatores, voir aussi ménestrels 32

joutes, voir aussi tournois $16,29^{23}, 35,42$, $45,47,89,161$

juridiction $9,20^{+33}, 42,45,48-49,53,55-$ $58,65,73,75-76,91^{54}, 101^{20}, 104-106$, 108-109, 111, 113, 116-117, 158

justice $9,11,20,35,38,50,56,74^{61}, 92$, $109,113^{+3}-114^{+4}, 116-119,121,138$, $153,161-163$

Kaysersberg $95^{3}-96$

Königreich, voir royaume d'Épiphanie

Königsegg 100

La Carité Nostre Dame des jogleors et des borgois, voir aussi confrérie des jongleurs et bourgeois 26

Lac de Constance 95, 111

Laon 86

lèpre, voir aussi maladrerie 88

Le Quesnoy 28-293 
Lille $10,16,20^{34}, 25,29,50-51,86,88-$ $89,91-92,136-138,161$

Limousin 150

Londres $25,40^{+92}$,

Lucerne $100-101,104^{29}, 107$

Lyon $39^{85}, 48,86,131$

Machelen 133

Mâcon 86

maïeur 26, 34

Maine 14-15

maître de cérémonie 40

majesté 9, 49, 159-160

maladrerie 88

malprofitants, voir abbé des malprofi tants

Mardi gras, voir aussi carnaval 11,118 , 132-133, 137-138

maréchaux, voir aussi roi des maréchaux $48,53,79$

Mayence $31,36,98,107^{+33}, 109-111$

mendiants, voir aussi roi des mendiants $20^{33}, 80,99^{14}, 147^{+6}, 152$

ménestrels, voir aussi roi des ménestrels $24,26^{+14}-33^{+51}, 37-41,43,47,89,95^{+3}$, 98-101, 111-112

merciers, voir aussi roi des merciers 14

messagers $37,75-78$

Metz 86

milieu 10-11, 18-19, 24, 84, 90-91, 113, 161

Minnesänger (troubadour) 30, 43

Mock kings, princes, voir rois moqueurs monde arabe 157

Mulhouse $95^{3}, 103$

musique $11^{+10}, 24,31^{+44}, 33-34,38-39$, 41,128

Namur $48,90-91^{50}$

Naples 31

Nîmes $15^{19}$

noblesse, voir aussi sociétés nobiliaires $44-45,48,53,56-57^{+67,70}, 58,136,164$

Normandie $15^{22}, 39,84,141,150$

Norvège 9,69

Noyon $85-86$

Nuitonie 100

office d'armes, voir aussi roi d'armes 32$33^{51}, 44,47,49-54^{+55,56}, 55,57^{67}-58$, $64,66^{+12}, 68^{+25}-69,71,74-78,161$

Ooidonk 133 ordre $15^{22}, 19,21,48,57,69,75,77^{+83}$ $78,88,93,99^{12}, 106,108^{37}, 135,161-$ 162

ordres chevaleresques (voir aussi Jarretière; Toison d'or) $15^{22}, 44-47,51,64^{+5}$ ordonnances $32,51-52^{+44}, 53,57^{70}, 83-$ $85^{+21}, 87,96,100,103,108,110-111$, $114,134,138,147$

- du 5 mars $149744-45,49-51,53-55^{+59}$, $56^{+64}, 58$

- du mois d'août 144814

origine (hypothèses d') 129

Orléans 85

Pape des Gingeans (société joyeuse à Lille) 137

Pâques (période de) 18

parenté sociale 38

Paris $12^{14}, 14-15,26^{+14}-27,32,35-38$, $87^{+32}, 90,113,117,119,128,147,150$ $151^{+25}, 152-153,163$

parlement de Paris 113, 117, 119

paroisse, voir aussi roi de la paroisse 89

Pays-Bas $44-46,49^{29}, 52-55,57-58,108$, 163

Pays de Bade 100, 104

père conduiseur 148

Péronne 89

Peu de Sens (société joyeuse à Lille) 137

Picardie 89, 141

Poitiers, bataille de 157

Poitou 150

Portugal 9, 69

précédence $74,77-78,141$

prérogatives $13,17,24,33,45,49,52-53$, $55,57,110-111,164$

prévôt (Voigt) 20, 26, 32, 85, 100

prévôt de mendiants (Bettelvoigt) $20^{33}$

prince $16,24-27,29,31,34,36-38,40$, $43,48-49,53^{+52}-54^{+55}, 55-57^{+70}, 64^{3}$, $67,71,81,84,86,126,131-132,134$, $138-139,142,146,155-156,162-164$

- d'amour (Aix-en-Provence) 17, 141

- de la jeunesse (Tournai) 17

- des chambres des rhétoriqueurs 140 144,163

- des puys mariales 141

- des sociétés joyeuses $11,17,136-137$

- des sots 25,138

- du mal-espargne (Abbeville) 17

privilèges $11,13-14,18,20,24,50,52$, $55,58,73,75-76,113,161$

procurateur $68^{25}, 73$ 
prostitution $80-83,86-87,90-91,93,96^{4}$, 161,163

protecteur $29-30,34,37-38,40^{92}, 83^{18}$, $100-103,108$

Provence $49^{30}, 53$

provinces (des rois d'armes) $15^{22}, 48,52$, $56^{+64}-57^{67,70}$

pseudo-dignitaire 133, 162

Pui de Amours (Tournai) 140

Puys poétiques, marials 25-26, 141

Quaëdypre 133

quartier, voir aussi roi du quartier $57^{70}, 80$, $87^{33}$

Quercy $15^{19}$

Ratsamhausen 100

Ravensburg $106^{31}, 108$

reinages $18^{29}$

rémunérations, voir aussi gages; gratifications 29, 37

Restor du paon (roman de Jean le Couret, dit Brisebarre) 41

rex flaiotelus, voir aussi roi des ménestrels $32^{+48}$

Rheinfelden 103, 105

Rheinische Knechtsordnung 110

ribauds/ribaudes, voir aussi roi des ribauds; prostitution $81^{+8,9}-82^{+14}, 83^{+18}, 85,91$, 93

Ribeauvillé (Rappoltsweiler) 99

rituel $17,34,41,77,93,102,110-112$, $119,124-130,133,139,144,156,162$

roi

- d'armes $15^{+22}, 20^{34}, 44^{+2}-48^{+24}, 49$ $52^{+48}, 53-57^{+67}, 58,64^{+5}-66^{+13}, 67-68$, 70-79, 161-164

- de France 9, 14-15 $5^{22}, 18^{28}, 20,35-38$, $46,80,84,90,163-164$

- ses doubles 157

- de la basoche $113-114,116^{+10}-119,121$, 162-163

- de l'abbaye, aussi dit Le grand abbé (Grenoble) 17

- de l'aumône 10

- de la Coquille 146, 148, 153

- de la fève (Bohnenkönig) 10, 12, 17-18, $119,124,126,128-130,138,161-163$

- de la paroisse $10,12,18^{+29}$

- de la rue 10

- de l'Épinette (Lille) $10-11^{+9}, 16^{+24}, 20^{34}$, $89,136,161$

- de professions $12-13,15,20,23$
- de Thunes 153,162

- des archers $10,16,20^{34}, 161,163$

- des barbiers 10

- des brigands 146

- des chaudronniers $10,20^{33}, 101$

- des clercs 10

- des compagnons 10, 15, 95-96, 103, 105-108, 110-112, 162, 164

- des couleuvriniers $20^{34}$

- des écoliers 10, 18

- des fous $11,33^{51}$

- des hérauts, voir aussi roi d'armes 10 , $12,15,32-33,35-37,40-41,44-48,58$, 64

- des lours (Arras) 17

- des maréchaux 33,48

- des mendiants 10,147-148, 161

- des ménestrels $10,13^{+15}-15,20^{33}, 24$ $35^{+62}, 36-43,45,47161,163-164$

- des merciers $10,12^{11}-14^{+16}, 15^{19}, 151^{25}$

- des ribauds $10,13^{+15}, 15,20^{33,34}, 32,80$ $81^{+6,9,82}, 83^{+16}-84^{+20}, 85^{+21}-86^{+29}, 87^{+32}-$ 93, 161-163

- des sociétés nobiliaires 15-16, 104

- du coq 10, 18

- du jeu d'échecs 155-156, 159-160

- du quartier 18

romans arthuriens 158

Rome 69,72

rois moqueurs (mock-kings, -princes) 131 rois festifs $12^{14}-13,15,17,123,162-163$

rois rituels $17-18,162-163$

Rottweil 107

Rouen 17, 25, 56, 131, 139

Rouergue $15^{19}$

Rouffach 103, 109

royaume $9-10,12^{14}, 15,24,32-33,36-37$, $39,44,48,53-54,64-65,68,70,74,76$, $79,81,93,95-96^{+4}, 98-99^{+13}, 100-$ $101^{+21}, 102^{+25}-103^{+26}, 104,106^{31}-108$, $110-113^{+2}, 114,116-117,146,148$, 153, 161, 163

- d'Épiphanie (Königreich) 102²5, 124 125, 127-130

- de jeunesse 11

royauté imaginaire 24,163

rue, voir aussi roi de la rue $132-134$

sacre 9

Saint-Antoine-le-Petit (église à Paris) 55, 57

Saint-Donat 10

Saint-Quentin 89 
Saturnales 129-130

Savoie $15^{19}, 39,49^{30}, 54^{54}$

sceaux $33-34,65^{+8}, 69,87,95-96^{+6}, 98^{9}-$ $99,111,134,159,163-164$

Schultheiss, voir écoutète

Sélestat 953 $, 103-104,108-109$

sergents $86^{+27}, 88,92$

sociétés $10-11,19-21,24-25,42,80-$ $82^{14}, 93,96,101-102^{25}, 113,147,151-$ $153,155,157,159,162$

- joyeuses $11-12^{14}, 16-17,83,89,131-$ $132,136-139,144,151^{23}$

- nobiliaires, voir aussi roi des sociétés nobiliaires $15^{+20}$

sots, voir aussi prince des sots 92,138

souverain, souveraineté $9,46,51,54,59$, $114,116-117,143$

Spielgraf, voir comte des ménestrels

statut royal $65,67-68,76,79$

Strasbourg 103, 108-111

Straubing $30,37,40$

subversion 16

Suisse $95^{+3}, 98-100,104,110$

symbole, symbolique $9,64,118,130,132$, $139,142-143,155,159$

taxes $38,69,87$

territoire $9,24,36-37,42,44,49,55,57$, $95^{3}, 100-101,134$

théâtre $11,107,113^{1}-114,119,136-138$ titre (dignité) $10-12^{+11,13}, 13,15-17,19-$ $20^{+33}, 24-25,27,29-38,40,43,49,54$, $64,67,73,79,82,89,93,98,116^{10}$, $134,136,142-143$
Toison d'or (ordre de la) $15^{22}, 44-47,49-55$

Toscane 82

Toulouse 86, 91, 119

Touraine 14-15

Tournai $17,25,29,46,86,91-92,140$, 164

tournoi $11^{9}, 16,44-45,56,89$

trompettiste $30^{34,35}-31,33$

Troyes 36

tuekien (tueur des chiens) 92

typologie $12-13,24,162$

Uechtland, voir Nuitonie

Uzès $15^{19}$

vagabonds $80-81^{9}, 89,93,161$

Valenciennes $25,28-29^{+23}, 45,86,139$

Veurne 133

Vienne $20^{33}, 26,35,50-51$

villes, alliance des (Städtebünde) 95

violence $82,109,112,146,151$

visitation $14,51,53,56,57^{+67}, 73,102^{25}$

Voigt, voir prévôt

voleurs $12^{14}, 84,147-148,151-153$

Vosges 98,100

Warhem 133

Weibel, voir huissier

Ypres 137, 140, 143

Zeeland 48

Zurich 95-96 $, 99-101,103-104^{+29}, 105-$ $106^{+31}, 107,111,164$ 